PSS PROCEEDINGS

\title{
Index and overlap construction for staggered fermions
}

\section{David H. Adams*}

Division of Mathematical Sciences, Nanyang Technological University, Singapore 637371

E-mail: dhadams@ntu.edu.sg

Recent developments regarding index and overlap construction for staggered fermions are reviewed, highlighting the surprising and unexpected aspects.

The XXVIII International Symposium on Lattice Field Theory

June 14-19,2010

Villasimius, Sardinia Italy

${ }^{*}$ Speaker. 


\section{Introduction}

Staggered fermions had long been perceived as disadvantaged compared to Wilson fermions regarding the index theorem connection between (would-be) zero-modes and gauge field topology. For Wilson fermions, the would-be zero-modes can be identified as eigenmodes with low-lying real eigenvalues; these can be assigned chirality \pm 1 according to the sign of $\bar{\psi} \gamma_{5} \psi$, thereby determining an integer-valued index which coincides with the topological charge of the background lattice gauge field in accordance with the index theorem when the gauge field is not too rough $[1,2,3]$. It coincides with the index obtained from the exact chiral zero-modes of the overlap Dirac operator [4]. In contrast, for staggered fermions, no way to identify the would-be zero-modes was known. They appeared to be mixed in with the other low-lying modes (all having purely imaginary eigenvalues) [1,5] and only separating out close to the continuum limit [6]. It seemed that, away from the continuum limit, the best one could have was a field-theoretic definition of the staggered fermion index [1]. The latter had the disadvantages of being non-integer, requiring a renormalization depending on the whole ensemble of lattice gauge fields, and being significantly less capable than the Wilson fermion index of maintaining the index theorem in rougher backgrounds [1].

Recently the consensus viewpoint described above was found to be incorrect: Staggered fermions do have identifiable would-be zero-modes away from the continuum limit, with identifiable chiralities and integer-valued index satisfying the index theorem when the lattice gauge field is not too rough [7]. The would-be zero-modes, chiralities and index can be identified in a spectral flow approach based on a new hermitian version of the staggered Dirac operator, paralleling the spectral flow approach to the index for usual Wilson fermions [2, 3].

Further developments along this line have led to a new version of overlap fermions built from staggered fermions in place of Wilson fermions [8]. The construction has the remarkable feature of reducing the 4 fermion flavors described by the staggered fermion to 2 flavors for the staggered overlap fermion. It turns out that underlying this construction is a new Wilson-type fermion, obtained by adding a Wilson-type term to the staggered fermion, which gives masses $\sim 1 / a$ to 2 of the flavors while leaving the remaining 2 flavors massless. Other Wilson-type terms are also possible; another one which reduces the flavors from 4 to 1 has subsequently been proposed in [9]. Numerical investigations of the 2-flavor staggered overlap fermion have been reported in [10].

A posteriori, these results and constructions can superficially seem quite straightforward. But a priori the odds were very much against any of this working out in a sensible way. There were a number of surprises and unexpected aspects, and these will be highlighted in the present review.

\section{Would-be zero-modes and index of the staggered Dirac operator}

In the continuum setting, the spectral flow perspective on the index of the Dirac operator $D$ arises by considering the eigenvalues $\{\lambda(m)\}$ of the hermitian operator

$$
H(m)=\gamma_{5}(D-m)
$$

The spectral flow is defined as the net number of eigenvalues $\lambda(m)$ of $H(m)$ that cross the origin, counted with sign \pm depending on the slope of the crossing, as $m$ is varied over some range. It can 
be shown that the spectral flow of $H(m)$ comes entirely from eigenvalue crossings at $m=0$ and equals minus the index of $D$.

In the lattice setting with Wilson fermions, the spectral flow perspective $[2,3]$ is based on the hermitian lattice analogue of (2.1):

$$
H_{W}(m)=\gamma_{5}\left(D_{W}-m\right)
$$

where $D_{W}$ is the Wilson Dirac operator. The eigenvalue crossings of $H_{W}(m)$ are in one-to-one correspondence with real eigenvalues of $D_{W}$, and the index of $D_{W}$ (obtained from the wouldbe zero-modes, i.e. the eigenmodes with low-lying real eigenvalues) coincides with minus the spectral flow of the low-lying eigenvalue crossings of $H_{W}(m)$. Numerical results illustrating this can be found, e.g., in [2]. An illustration in the $d=2$ case is given in Fig. 1 where the eigenvalues of $H_{W}(m)$ are plotted as functions of $m$.

In the case of staggered fermions, the staggered Dirac operator $D_{s t}$ is anti-hermitian and therefore all its eigenvalues are purely imaginary. Hence the identification of would-be zero modes and index in the Wilson case does not carry over to the staggered case: there are no real eigenvalues, and in fact the staggered analogue of $(2.2), \Gamma_{5}\left(D_{s t}-m\right)$, is not even hermitian. The lack of any obvious way to distinguish the would-be zero-modes from the other low-lying eigenmodes of $D_{s t}$ gave rise to the consensus viewpoint that staggered fermions are disadvantaged in this regard relative to Wilson fermions.

However, it turns out that there is an alternative spectral flow approach in the staggered case [7]. Note that in the continuum setting, instead of (2.1) one can just as well use the hermitian operator $H(m)=i D-m \gamma_{5}$ for the spectral flow perspective on the index. But now the staggered analogue,

$$
H_{s t}(m)=i D_{s t}-m \Gamma_{5}
$$

is also hermitian and so its spectral flow can be considered as well. Here $\Gamma_{5}$ is the analogue of $\gamma_{5}$ in the staggered formulation; it is hermitian and corresponds up to $O\left(a^{2}\right)$ discretization errors to $\gamma_{5} \otimes 1$ in the $\operatorname{spin} \otimes$ flavor interpretation [11]. Since $H_{s t}(0)=i D_{s t}$, the would-be zero-modes of $D_{s t}$ are able to be identified as the eigenmodes with eigenvalues $-i \lambda=-i \lambda(0)$ for which the associated flow $\lambda(m)$ crosses zero at a low-lying value of $m$. Furthermore, the sign of the slope of the crossing is minus the chirality of the would-be zero-mode, and hence the index is minus the spectral flow of $H_{s t}(m)$ coming from the crossings at low-lying values of $m$. See [7] for the details of this identification.

This way of identifying the would-be zero-modes of $D_{s t}$ from the low-lying eigenvalue crossings of $H_{s t}(m)$ relies on an implicit assumption, namely that there is a clear separation between the low-lying and high-lying crossings. Actually, there is no a priori reason to believe that this assumption is true, even in smooth gauge field backgrounds or in the free field case. In fact one would expect that it is not true. The clear separation between low-lying and high-lying crossings in the Wilson case (as seen in Fig.1) relies crucially on the property $\gamma_{5}^{2}=\mathbf{1}$. But the staggered version $\Gamma_{5}$ does not have this property. The eigenvalues of $\Gamma_{5}$ are not \pm 1 but are distributed throughout the interval $[-1,1]$. E.g. 0 is an eigenvalue of $\Gamma_{5}$ in the free field case; this can be seen from the free field momentum representation of $\Gamma_{5}^{2}$ which is $\prod_{v} \cos ^{2}\left(p_{v}\right)$. In light of this one would expect 
that, even in the free field case, the eigenvalue crossings of $H_{s t}(m)$ will be an arbitrary mess with no clear separation into low-lying and high-lying crossings.

The first and biggest surprise in all this - a miracle in fact - is that, contrary to expectations, the spectral flow of $H_{s t}(m)$ does have a clear separation between low-lying and high-lying eigenvalue crossings, at least when the gauge field is not too rough. In fact in the free field case there are no high-lying crossings at all, cf. the bound (2.7) below. Fig.2 shows the spectral flow in a moderately roughened $\mathrm{U}(1)$ background with topological charge $Q=1$ on a 2-dimensional lattice. Now there are high-lying crossings, but they are clearly separated from the low-lying ones, so the would-be zero-modes of $D_{s t}$, their chiralities, and index, can be unambiguously identified.

The absence of high-lying eigenvalue crossings for $H_{s t}(m)$ in the free field case can be seen analytically as follows. A simple calculation of $H_{s t}(m)^{2}$ in the free field momentum representation gives

$$
\hat{H}_{s t}(m)^{2}=\sum_{\mu} \sin ^{2}\left(p_{\mu}\right)+m^{2} \prod_{v} \cos ^{2}\left(p_{v}\right)
$$

Set $s_{\mu}=\sin \left(p_{\mu}\right), c_{v}=\cos \left(p_{v}\right)$. Then, in the case of 2 spacetime dimensions, starting from $\hat{H}_{s t}(m)^{2}=s_{1}^{2}+s_{2}^{2}+m^{2}\left(1-s_{1}^{2}\right)\left(1-s_{2}^{2}\right)$, we find

$$
\hat{H}_{s t}(m)^{2}=m^{2}+\left(1-m^{2}\right)\left(s_{1}^{2}+s_{2}^{2}\right)+m^{2} s_{1}^{2} s_{2}^{2} \quad \geq m^{2} \quad \text { for } 0 \leq|m| \leq 1
$$

and

$$
\hat{H}_{s t}(m)^{2}=1+s_{1}^{2} s_{2}^{2}+\left(m^{2}-1\right)\left(1-s_{1}^{2}\right)\left(1-s_{2}^{2}\right) \quad \geq 1 \quad \text { for }|m| \geq 1
$$

Note that both of these bounds are saturated. Identical bounds can be derived in the $d=4$ case, although the derivations are more complicated. Hence in the free field case, for both $d=2$ and $d=4$ dimensions (and probably also for higher dimensions), we have

$$
H_{s t}(m)_{\text {free }}^{2} \geq \begin{cases}m^{2} & \text { for }|m| \leq 1 \\ 1 & \text { for }|m| \geq 1\end{cases}
$$

This bound has a generalization to the case of gauge fields satisfying an "admissibility condition" on the plaquettes. It shows a separation between low-lying and high-lying eigenvalue crossings for $H_{s t}(m)$ when the $\varepsilon$ in the condition is sufficiently small - see [7] for details. Thus the situation is analogous to the Wilson case where the admissibility condition guarantees the separation between low-lying and high-lying crossings for the hermitian Wilson operator $H_{W}(m)$ [12].

Comparing Fig.'s 1 and 2 we see that the form of the spectral flow of $H_{s t}(m)$ is very different from the Wilson case, and the separation between the low-lying and high-lying crossings is much larger. However, this is not one of the surprises alluded to in the abstract. Instead, the surprise here is that there is no surprise - the staggered spectral flow has the same form as in the Wilson case once the correct interpretation of the hermitian staggered operator $H_{s t}(m)$ is identified. It turns out that the parameter $m$ in the staggered case should be identified not with the corresponding $m$ in $H_{W}(m)$ but with the Wilson parameter $r$ in the Wilson case. This will be explained further below; see Fig.3. 
Since the staggered fermion in $d$ dimensions describes $2^{d / 2}$ flavors, the index theorem in this case should be index $\left(D_{s t}\right)=2^{d / 2}(-1)^{d / 2} Q$. This is confirmed by numerical results in smooth enough backgrounds. E.g. in Fig. 2 the two positive slope low-lying crossings in the $Q=1$ background in 2 dimensions imply that the index is -2 in accordance with the index theorem. The eigenvalues of $D_{s t}$ in this background correspond to $m=0$ in Fig.2. The would-be zero-modes of $D_{s t}$ can be identified as the two eigenmodes with the low-lying eigenvalues belonging to the two eigenvalue flows that cross the origin.

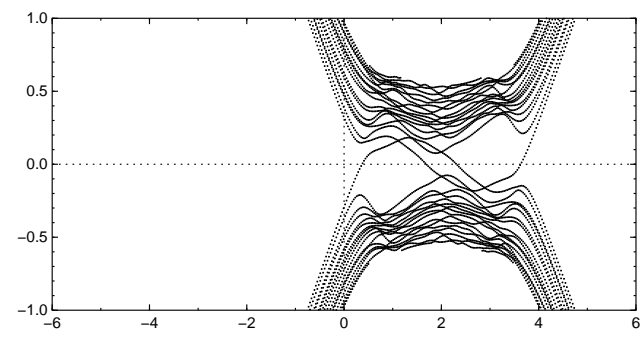

Figure 1: Eigenvalue flow of $H_{W}(m)$ in a $Q=1$ background on a 2 -dim lattice.

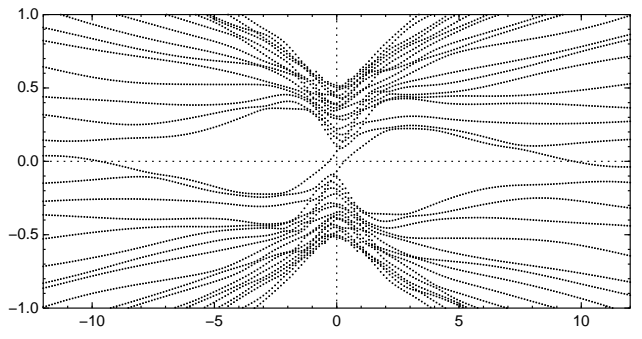

Figure 2: Eigenvalue flow of $H_{s t}(m)$ in the same $Q=1$ background as Fig. 1 .

\section{Staggered overlap construction}

In the Wilson case, the spectral flow perspective on the index leads to

$$
\operatorname{index}\left(D_{W}\right)=-\frac{1}{2} \operatorname{Tr} \frac{H_{W}\left(m_{0}\right)}{\sqrt{H_{W}\left(m_{0}\right)^{2}}}=\operatorname{index}\left(D_{o v}\right)
$$

for any $m_{0}$ in the region between where the low-lying and high-lying eigenvalue crossings of $H_{W}(m)$ occur (e.g. $\left.m_{0}=1 / a\right)[3,4]$. Here $D_{o v}=\frac{1}{a}\left(1+\gamma_{5} \frac{H_{W}\left(m_{0}\right)}{\sqrt{H_{W}\left(m_{0}\right)^{2}}}\right)$ is the overlap Dirac operator [4]. The intimate connection between the Wilson index, the hermitian operator $H_{W}(m)$ and the overlap Dirac operator suggests there may exist a staggered version of the overlap Dirac operator connected to the staggered index and staggered hermitian operator $H_{s t}(m)$ discussed above. But there is a problem: The connections and properties of the overlap Dirac operator in the Wilson case rely crucially on the property $\gamma_{5}^{2}=\mathbf{1}$. (E.g. without it the GW relation would not hold and $D_{o v}$ would not have exact zero-modes.) Therefore the natural replacement $\gamma_{5} \rightarrow \Gamma_{5}$ is not possible when constructing the overlap operator in the staggered case since $\Gamma_{5}^{2} \neq \mathbf{1}$. Attempts to construct different versions of $\gamma_{5}$ in the staggered case which do satisfy $\gamma_{5}^{2}=\mathbf{1}$ invariably lead to unnatural, problematic operators which violate either lattice rotation invariance or gauge invariance [13].

This " $\Gamma_{5}^{2} \neq \mathbf{1}$ problem" initially appears insurmountable, but the second main surprise in all this is that it does have a solution [8]. The theoretical idea behind the solution is as follows. In the staggered setting there is a naturally arising operator which squares to the identity, namely $\Gamma_{55}$, acting on the staggered fermion fields by $\Gamma_{55} \chi(x)=(-1)^{n_{1}+\ldots+n_{d}} \chi(x)$. It has the spin $\otimes$ flavor interpretation $\gamma_{5} \otimes \gamma_{5}$, which is not what we want. But if the staggered overlap construction can be set up such that the physical flavors are those with positive flavor-chirality under $\mathbf{1} \otimes \gamma_{5}$ then $\gamma_{5} \otimes \gamma_{5}$ will be the same as $\gamma_{5} \otimes \mathbf{1}$ on the physical flavors, and then $\Gamma_{55}$ may be used for the role of $\gamma_{5}$ in the staggered overlap construction. 
In fact this can be achieved simply by replacing $\gamma_{5} \rightarrow \Gamma_{55}$ and $H_{W} \rightarrow H_{s t}$ in the overlap formula for $D_{o v}$. The key observation is that in this construction we have $\Gamma_{55} H_{s t}\left(m_{0}\right)=i \Gamma_{55} D_{s t}-m_{0} \Gamma_{55} \Gamma_{5}$ and $\Gamma_{55} \Gamma_{5}$ has the spin $\otimes$ flavor interpretation $\left(\gamma_{5} \otimes \gamma_{5}\right)\left(\gamma_{5} \otimes \mathbf{1}\right)=\mathbf{1} \otimes \gamma_{5}$ up to $O\left(a^{2}\right)$ effects. Thus the 2 fermion flavors with positive flavor-chirality get a negative mass from the $-m_{0} \Gamma_{55} \Gamma_{5}$ term, and hence become massless flavors of the staggered overlap fermion, while the 2 flavors with negative flavor-chirality get positive masses from this term and hence become heavy, decoupling flavors of the staggered overlap fermion with masses $\sim 1 / a$, just like the "doubler" species in the usual overlap fermion construction. Note that the exact flavored chiral symmetry $\left\{D_{s t}, \Gamma_{55}\right\}=0$ of the staggered fermion hereby becomes an exact unflavored GW chiral symmetry $\left\{D_{\text {sov }}, \Gamma_{55}\right\}=$ $a D_{s o v} \Gamma_{55} D_{\text {sov }}$ of the resulting staggered overlap Dirac operator $D_{s o v}$. Moreover, a staggered version of the index relations (3.1) holds [7, 8]: $\frac{1}{2} \operatorname{index}\left(D_{s t}\right)=-\frac{1}{2} \operatorname{Tr} \frac{H_{s t}\left(m_{0}\right)}{\sqrt{H_{s t}\left(m_{0}\right)^{2}}}=\operatorname{index}\left(D_{s o}\right)$. The factor $\frac{1}{2}$ multiplying index $\left(D_{s t}\right)$ reflects the reduction from 4 to 2 flavors in the staggered overlap fermion.

The interpretation of the staggered overlap fermion becomes more straightforward if we change the hermitian staggered operator by $H_{s t}(m) \rightarrow \Gamma_{55} D_{s t}-m \Gamma_{5}=\Gamma_{55}\left(D_{s t}-m \Gamma_{55} \Gamma_{5}\right)$. As mentioned in [8], this operator is closely related to, and has the same eigenvalue spectrum as, the previous operator $i D_{s t}-m \Gamma_{5}$. Everything in the preceding continues to hold with this new $H_{s t}(m)$. The staggered overlap Dirac operator takes a more recognizable form though: it can now be written as

$$
D_{s o v}=\frac{1}{a}\left(1+\left(D_{s t}-m_{0} \Gamma_{55} \Gamma_{5}\right) \frac{1}{\sqrt{\left(D_{s t}-m_{0} \Gamma_{55} \Gamma_{5}\right)^{\dagger}\left(D_{s t}-m_{0} \Gamma_{55} \Gamma_{5}\right)}}\right)
$$

From this we see that underlying the staggered overlap construction is a new staggered version of Wilson fermions with the Dirac operator

$$
D_{s W}=D_{s t}+W_{s t} \quad, \quad W_{s t}=\frac{r}{a}\left(1-\Gamma_{55} \Gamma_{5}\right) .
$$

The "Wilson term" $W_{s t}$ decouples the negative flavor-chirality modes by giving them mass $2 r / a$ while keeping the two positive flavor-chirality modes as the physical modes. Hence $D_{s W}$ describes two physical quark flavors on which $\Gamma_{5}=\Gamma_{55}$ up to $O(a)$ effects. It has the $\Gamma_{55}$ hermiticity $D_{s W}^{\dagger}=$ $\Gamma_{55} D_{s W} \Gamma_{55}$. A 2-flavor overlap fermion can then be obtained by taking $D_{s W}-m$ with $m=\frac{r \rho}{a}$, $\rho \in(0,2)$ as the kernel in the usual overlap construction. For $\frac{r}{a}=m_{0}$ and $\rho=1$ this is precisely the 2-flavor staggered overlap Dirac operator $D_{\text {sov }}$ obtained above in (3.2). But now we see that it can be generalized to any $\rho \in(0,2)$. Furthermore, the role of the parameter $m_{0}$ in the staggered overlap construction is hereby clarified: it is analogous to the Wilson parameter in the usual overlap construction.

The general staggered overlap operator can also be expressed as $D_{s o v}=\frac{1}{a}\left(1+\Gamma_{55} \frac{H_{s W}(\rho)}{\sqrt{H_{s W}(\rho)^{2}}}\right)$ where $H_{s W}$ is another hermitian staggered operator given by ${ }^{1}$

$$
H_{s W}(m)=\Gamma_{55}\left(D_{s W}-m\right)=\Gamma_{55} D_{s t}-\Gamma_{5}+(1-m) \Gamma_{55}
$$

This is the true analogue of the hermitian Wilson operator $H_{W}(m)=\gamma_{5}\left(D_{W}-m\right)$, and its spectral flow has a similar form (Fig.4). ${ }^{2}$ On the other hand, the spectral flow of the hermitian Wilson

\footnotetext{
${ }^{1}$ We set $r=1$ and use lattice units to get the second equality.

${ }^{2}$ In 2 dimensions the staggered Wilson fermion has one physical flavor and one doubler whereas the usual Wilson fermion has three doublers. This explains why there is one high-lying eigenvalue crossing in Fig.4 and three high-lying crossings in Fig.1.
} 
operator as a function of the Wilson parameter $r$, with fixed $m=1$ (Fig.3), has a similar form to the spectral flow of our previous hermitian staggered operator $H_{s t}(m)$ as a function of $m$ (Fig.2) as anticipated. ${ }^{3}$

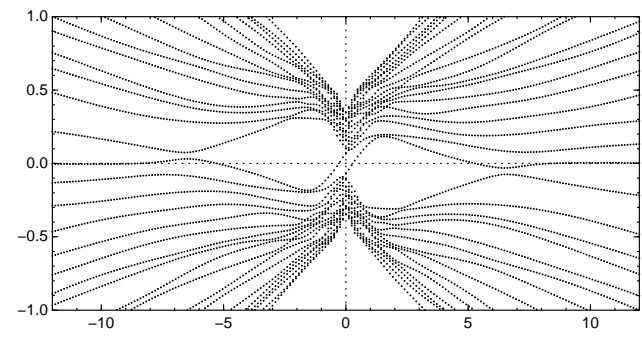

Figure 3: Eigenvalue flow of $H_{W}(m=1)$ as a function of the Wilson parameter $r$.

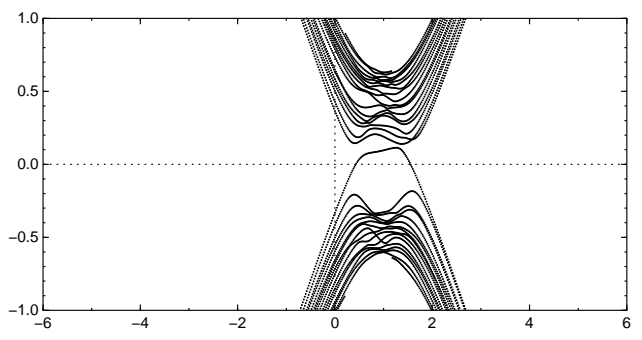

Figure 4: Eigenvalue flow of $H_{s W}(m)$ in the same $Q=1$ background as Fig.s 1,2,3.

To summarize, three new lattice fermion formulations have been introduced: staggered versions of Wilson fermions, domain wall fermions ${ }^{4}$ and overlap fermions. Each of these is a new alternative and competitor to the corresponding Wilson-based formulation, and should be more computationally efficient since the constructions start from staggered rather than naive fermions. However, the gain in efficiency is reduced because the staggered "Wilson term" is less local; it involves $\Gamma_{5}$ which is a 4-link operator [10]. This reduction in efficiency may possibly be ameliorated by smearing the links [9].

\section{References}

[1] J. Smit and J.C. Vink, Nucl. Phys. B 286, 485 (1987)

[2] S. Itoh, Y. Iwasaki and T. Yoshié, Phys. Rev. D 36, 527 (1987)

[3] R. Narayanan and H. Neuberger, Nucl. Phys. B 443, 305 (1995)

[4] H. Neuberger, Phys. Lett. B 417, 141 (1998); ibid 427, 353 (1998)

[5] P.H. Damgaard, U.M. Heller, R. Niclasen and K. Rummukainen, Phys. Rev. D 61:014501 (1999)

[6] E. Follana, A. Hart and C.T.H. Davies, Phys. Rev. Lett. 93:241601 (2004)

[7] D.H. Adams, Phys. Rev. Lett. 104:141602 (2010)

[8] D.H. Adams, arXiv:1008.2833

[9] C. Hoelbling, Phys. Lett. B 696, 422 (2011)

[10] Ph. de Forcrand, A. Kurkela and M. Panero, in proceedings of Lattice 2010 [arXiv:1102.1000]

[11] M.F.L. Golterman and J. Smit, Nucl. Phys. B 245, 61 (1984)

[12] P. Hernandez, K. Jansen and M. Lüscher, Nucl. Phys. B 552,363 (1999); H. Neuberger, Phys. Rev. D 61:085015, 2000

[13] D.H. Adams, unpublished calculations

\footnotetext{
${ }^{3}$ Note from (3.4) that the flow parameter $m$ of $H_{s W}(m)$ is multiplied onto $\Gamma_{55}$, whereas for $H_{s t}(m)$ it is multiplied onto $\Gamma_{5}$. Thus the gauge field topology can be probed by a hermitian operator whose varying term is the flavor nonsinglet $m \Gamma_{55}$, contrary to an incorrect claim in [10].

${ }^{4}$ Staggered domain wall fermions were not discussed here; see [8].
} 